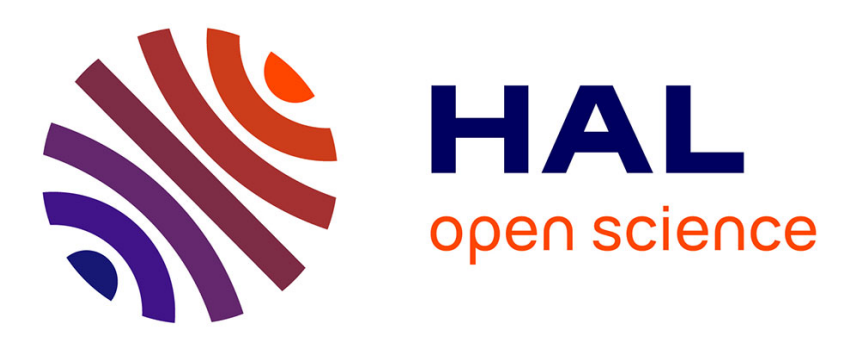

\title{
Large deviations for Gaussian stationary processes and semi-classical analysis
}

\author{
Bernard Bercu, Jean-François Bony, Vincent Bruneau
}

\section{To cite this version:}

Bernard Bercu, Jean-François Bony, Vincent Bruneau. Large deviations for Gaussian stationary processes and semi-classical analysis. Séminaire de Probabilités, 2012, 44, pp.409-428. 10.1007/9783-642-27461-9 . hal-00642658

\section{HAL Id: hal-00642658 \\ https://hal.science/hal-00642658}

Submitted on 23 Nov 2011

HAL is a multi-disciplinary open access archive for the deposit and dissemination of scientific research documents, whether they are published or not. The documents may come from teaching and research institutions in France or abroad, or from public or private research centers.
L'archive ouverte pluridisciplinaire HAL, est destinée au dépôt et à la diffusion de documents scientifiques de niveau recherche, publiés ou non, émanant des établissements d'enseignement et de recherche français ou étrangers, des laboratoires publics ou privés. 
Noname manuscript No.

(will be inserted by the editor)

\title{
Large deviations for Gaussian stationary processes and semi-classical analysis
}

\author{
Bernard Bercu · Jean-François Bony · Vincent Bruneau
}

the date of receipt and acceptance should be inserted later

\begin{abstract}
In this paper, we obtain a large deviation principle for quadratic forms of Gaussian stationary processes. It is established by the conjunction of a result of Roch and Silbermann on the spectrum of products of Toeplitz matrices together with the analysis of large deviations carried out by Gamboa, Rouault and the first author. An alternative proof of the needed result on Toeplitz matrices, based on semi-classical analysis, is also provided.
\end{abstract}

Keywords Large deviations - Gaussian processes · Toeplitz matrices · Distribution of eigenvalues

\section{Introduction}

For any bounded measurable real function $f$ on the torus $\mathbb{T}=\left[-\pi, \pi\left[\right.\right.$, the $\ell^{2}(\mathbb{N})$ Toeplitz and Hankel operators are respectively defined as

$$
T(f)=\left(\widehat{f}_{i-j}\right)_{i, j \geq 0} \quad \text { and } \quad H(f)=\left(\widehat{f}_{i+j+1}\right)_{i, j \geq 0}
$$

where $\left(\widehat{f}_{n}\right)$ stands for the sequence of Fourier coefficients of $f$. We refer the reader to the books of Böttcher and Silbermann [3], [4] for a general presentation of Toeplitz operators. A well-known identity between the product $T(f) T(g)$ and $T(f g)$ is

$$
T(f g)-T(f) T(g)=H(f) H(\widetilde{g})
$$

where $\widetilde{g}(x)=g(-x)$. The analogue of identity (1.2) for finite section Toeplitz matrices is given by the formula of Widom [16]

$$
T_{n}(f g)-T_{n}(f) T_{n}(g)=P_{n} H(f) H(\widetilde{g}) P_{n}+Q_{n} H(\widetilde{f}) H(g) Q_{n}
$$

where the projection $P_{n}$ and the operator $Q_{n}$ are given by

$$
\begin{aligned}
P_{n}\left(x_{0}, x_{1}, x_{2}, \ldots\right) & =\left(x_{0}, x_{1}, \ldots, x_{n}, 0, \ldots\right), \\
Q_{n}\left(x_{0}, x_{1}, x_{2}, \ldots\right) & =\left(x_{n}, x_{n-1}, \ldots, x_{0}, 0, \ldots\right),
\end{aligned}
$$

B. Bercu · J.-F. Bony · V. Bruneau

Université Bordeaux 1, Institut de Mathématiques de Bordeaux,

UMR CNRS 5251, 351 cours de la libération, 33405 Talence cedex, France.

E-mail: Bernard.Bercu@math.u-bordeaux1.fr

E-mail: Jean-Francois.Bony@math.u-bordeaux1.fr

E-mail: Vincent.Bruneau@math.u-bordeaux1.fr 
and $T_{n}(f)$ is the finite section of order $n \geq 1$ of $T(f)$ which means that $T_{n}(f)$ is identified with $P_{n} T(f) P_{n}$. In other words, our operators will be considered as operators on $\operatorname{Im} P$ and $\operatorname{Im} P_{n}$ where $P$ stands for the projection operator on $\ell^{2}(\mathbb{N})$. We clearly have $Q_{n}^{2}=P_{n}, P_{n} Q_{n}=Q_{n} P_{n}=Q_{n}$, and $Q_{n} T_{n}(f) Q_{n}=T_{n}(\widetilde{f})$.

The classical Szegö theorem deals with the asymptotic behavior of the spectrum of a single Toeplitz matrix. It states that if $f$ is a bounded measurable real function on $\mathbb{T}$, the limiting set of eigenvalues of the sequence $\left(T_{n}(f)\right)$ is exactly

$$
\sigma(T(f))=[\operatorname{essinf} f, \operatorname{esssup} f]
$$

where $\sigma(T(f))$ denotes the spectrum of the operator $T(f)$. Moreover, the empirical spectral measure of $\left(T_{n}(f)\right)$ converges to $P_{f}$ which is the image probability of the uniform measure on $\mathbb{T}$ by the application $f$. In other words, if $\lambda_{0}^{n}, \ldots, \lambda_{n}^{n}$ are the eigenvalues of $T_{n}(f)$, then for any bounded continuous real function $\varphi$

$$
\lim _{n \rightarrow \infty} \frac{1}{n} \sum_{k=0}^{n} \varphi\left(\lambda_{k}^{n}\right)=\frac{1}{2 \pi} \int_{\mathbb{T}} \varphi(f(x)) d x
$$

In particular, the maximum eigenvalue of $T_{n}(f)$ converges to $\operatorname{esssup} f$ while the minimum eigenvalue of $T_{n}(f)$ converges to essinf $f$. One can find more details in Section 5.2 of [10] or in Section 5.4 of [4]. Our purpose is to make use of similar results for the spectrum of the product of two Toeplitz matrices $T_{n}(f) T_{n}(g)$. Several authors have investigated the asymptotic behavior of the spectrum of $T_{n}(f) T_{n}(g)$. More precisely, it was shown in Lemma 5 of [1] or Lemma 2.6 of [15] that if $f$ and $g$ are two bounded measurable real functions on $\mathbb{T}$, then the empirical spectral measure associated with the sequence $\left(T_{n}(f) T_{n}(g)\right)$ converges to the limiting measure $P_{f g}$. However, the limiting set of eigenvalues of $\left(T_{n}(f) T_{n}(g)\right)$ is much more difficult to understand. Via a theorem of Roch and Silbermann, we shall see that, as soon as $f$ and $g \geq 0$ are bounded piecewise continuous real functions, the limiting set of eigenvalues of $\left(T_{n}(f) T_{n}(g)\right)$ coincides with the spectrum of the limiting operator $T(f) T(g)$. In particular, the maximum and the minimum eigenvalues of $T_{n}(f) T_{n}(g)$ both converge to the maximum and minimum of the spectrum of $T(f) T(g)$.

In this paper, we make use of the previous results on Toeplitz operators to obtain a large deviation principle (LDP) for quadratic forms of Gaussian stationary processes. More precisely, consider a centered stationary real Gaussian process $\left(X_{n}\right)$ with bounded piecewise continuous spectral density $g$. It was shown in [1] an LDP for subsequences of the empirical periodogram $\left(\mathcal{W}_{n}(f)\right)$ integrated over a bounded piecewise continuous real function $f$. We can now deduce a full LDP for the sequence $\left(\mathcal{W}_{n}(f)\right)$.

We also give an alternative proof of the theorem of Roch and Silbermann in the particular case of Toeplitz operators with continuous symbols. Our approach is based on semi-classical analysis and scattering theory by construction of quasimodes which are approximative eigenvectors. We hope that this microlocal approach can be used in other situations.

The paper is organized as follows. In Section 2, we recall a theorem of Roch and Silbermann. Section 3 is devoted to the application in probability. An enlightening example is treated in Section 4. Then, we give our alternative proof of the result of Roch and Silbermann in the case of Toeplitz operators with continuous symbols. This result and our functional point of view on Toeplitz operators are given in Section 5. The convergence of the spectrum is proved in Section 6. Finally, in Section 7, we propose an alternative proof of Coburn's theorem dealing with the essential spectrum of products of Toeplitz operators. 


\section{Results on Toeplitz operators}

Denote by $\mathcal{A}$ the Banach algebra of all sequences $\left(A_{n}\right)$ of uniformly bounded linear operators on $\operatorname{Im} P_{n}$ endowed with the sum and the composition term by term, and the supremum of the operator norm of the elements. Let $\mathcal{B}$ be the collection of all sequences $\left(A_{n}\right)$ of $\mathcal{A}$ for which one can find two bounded linear operators $A$ and $\widetilde{A}$ in $\operatorname{Im} P$ such that

$$
A_{n} \rightarrow A, \quad A_{n}^{*} \rightarrow A^{*}, \quad Q_{n} A_{n} Q_{n} \rightarrow \widetilde{A}, \quad Q_{n} A_{n}^{*} Q_{n} \rightarrow \widetilde{A}^{*},
$$

where $*$ stands the adjoint operator and $\rightarrow$ stands for the strong convergence. Finally, denote by $\mathcal{C}$ the smallest closed subalgebra of $\mathcal{A}$ containing the collection of all sequences $\left(T_{n}(f)\right)$ where $f$ are bounded piecewise continuous real functions. In fact, $\mathcal{C}$ is a subalgebra of $\mathcal{B}$ and

$$
T_{n}(f) \rightarrow T(f), \quad Q_{n} T_{n}(f) Q_{n} \rightarrow T(\tilde{f}) .
$$

We refer to Section 2.5 of [4] for more details on $\mathcal{B}$. We are now in position to state a theorem of Roch and Silbermann.

Theorem 2.1 (Roch-Silbermann) Let $\left(T_{n}\right)$ be a sequence of selfadjoint operators of $\mathcal{C}$. Moreover, denote the strong limits of $T_{n}$ and $Q_{n} T_{n} Q_{n}$ by $T$ and $\widetilde{T}$, respectively. For $\lambda \in \mathbb{R}$, the following properties are equivalent:

i) $\lambda \in \sigma(T) \cup \sigma(\widetilde{T})$,

ii) $\lambda$ is the limit of a sequence $\left(\lambda_{n}\right)$ where $\lambda_{n} \in \sigma\left(T_{n}\right)$,

iii) $\lambda$ is the limit of a subsequence $\left(\lambda_{n_{k}}\right)$ where $\lambda_{n_{k}} \in \sigma\left(T_{n_{k}}\right)$.

Theorem 2.1 was established in [14] together with several examples of application. It is given, in its present form, in Theorem 4.16 of [4].

A direct application of this result is as follows. First of all, let us introduce some notations. Let $f$ and $g$ be two bounded piecewise continuous real functions with $g \geq 0$. From Lemma 6.1 below, the sequence $\left(T_{n}(g)^{1 / 2}\right)$ as well as $\left(T_{n}(g)^{1 / 2} T_{n}(f) T_{n}(g)^{1 / 2}\right)$ belong to $\mathcal{C}$,

$$
\begin{gathered}
T_{n}(g)^{1 / 2} T_{n}(f) T_{n}(g)^{1 / 2} \rightarrow T(g)^{1 / 2} T(f) T(g)^{1 / 2}, \\
Q_{n} T_{n}(g)^{1 / 2} T_{n}(f) T_{n}(g)^{1 / 2} Q_{n} \rightarrow T(\widetilde{g})^{1 / 2} T(\widetilde{f}) T(\widetilde{g})^{1 / 2} .
\end{gathered}
$$

On $\operatorname{Im} P_{n}$, we clearly have

$$
\sigma\left(T_{n}(f) T_{n}(g)\right)=\sigma\left(T_{n}(g)^{1 / 2} T_{n}(f) T_{n}(g)^{1 / 2}\right),
$$

with the same multiplicity. Moreover, by Lemma 6.6, we also have on $\operatorname{Im} P$

$$
\sigma\left(T(g)^{1 / 2} T(f) T(g)^{1 / 2}\right)=\sigma(T(f) T(g))=\sigma(T(\widetilde{f}) T(\widetilde{g}))=\sigma\left(T(\widetilde{g})^{1 / 2} T(\widetilde{f}) T(\widetilde{g})^{1 / 2}\right) .
$$

Denote the maximum and minimum eigenvalues of $T_{n}(f) T_{n}(g)$ by

$$
\begin{aligned}
\lambda_{\max }^{n}(f, g) & =\max \sigma\left(T_{n}(f) T_{n}(g)\right), \\
\lambda_{\min }^{n}(f, g) & =\min \sigma\left(T_{n}(f) T_{n}(g)\right) .
\end{aligned}
$$

In addition, denote the extrema of the spectrum of $T(f) T(g)$ by

$$
\begin{aligned}
\lambda_{\max }(f, g) & =\max \sigma(T(f) T(g)), \\
\lambda_{\min }(f, g) & =\min \sigma(T(f) T(g)) .
\end{aligned}
$$

One can observe that, in general, we do not know if $\lambda_{\max }(f, g)$ and $\lambda_{\min }(f, g)$ are eigenvalues. 
Corollary 2.2 Assume that $f$ and $g$ are two bounded piecewise continuous real functions on $\mathbb{T}$ with $g \geq 0$. Then, the limiting sets of eigenvalues of the sequence $\left(T_{n}(f) T_{n}(g)\right)$ are given by $\sigma(T(f) T(g))$. In particular,

$$
\begin{aligned}
& \lim _{n \rightarrow \infty} \lambda_{\max }^{n}(f, g)=\lambda_{\max }(f, g), \\
& \lim _{n \rightarrow \infty} \lambda_{\min }^{n}(f, g)=\lambda_{\min }(f, g) .
\end{aligned}
$$

In Section 4, we shall show via an example related to Gaussian autoregressive process that it is not true in general that for two bounded continuous real functions $f$ and $g, \lambda_{\max }(f, g)=\sup (f g)$ or $\lambda_{\min }(f, g)=\inf (f g)$. One can also observe that the norm of $T(g)^{1 / 2} T(f) T(g)^{1 / 2}$ is not always equal to $\|f g\|_{\infty}$ or $\|f\|_{\infty}\|g\|_{\infty}$. The situation is totally different from the case of a single Toeplitz operator $T(f)$ with bounded continuous real function as $\lambda_{\max }(f, 1)=\sup (f)$ and $\lambda_{\min }(f, 1)=\inf (f)$.

\section{Application in probability}

Let $\left(X_{n}\right)$ be a centered stationary real Gaussian process with bounded piecewise continuous spectral density $g \geq 0$ which means that

$$
\mathbb{E}\left[X_{j} X_{k}\right]=\frac{1}{2 \pi} \int_{\mathbb{T}} \exp (i(j-k) x) g(x) d x
$$

We assume in all the sequel that $g$ is not the zero function. For any bounded piecewise continuous real function $f$ on the torus $\mathbb{T}$, we are interested in the asymptotic behavior of

$$
\mathcal{W}_{n}(f)=\frac{1}{2 \pi n} \int_{\mathbb{T}} f(x)\left|\sum_{j=0}^{n} X_{j} \exp (i j x)\right|^{2} d x
$$

The purpose of this section is to provide the last step in the analysis of the large deviation properties of $\left(\mathcal{W}_{n}(f)\right)$ by establishing an LDP for $\left(\mathcal{W}_{n}(f)\right)$ in the spirit of the original work of [1] or of Bryc and Dembo [6]. We refer the reader to the book of Dembo and Zeitouni [7] for the general theory on large deviations. The covariance matrix associated with the vector $X^{(n)}=\left(X_{0}, \ldots, X_{n}\right)^{t}$ is $T_{n}(g)$. Consequently, it immediately follows from (3.1) that

$$
\mathcal{W}_{n}(f)=\frac{1}{n} X^{(n) t} T_{n}(f) X^{(n)}=\frac{1}{n} Y^{(n) t} T_{n}(g)^{1 / 2} T_{n}(f) T_{n}(g)^{1 / 2} Y^{(n)}
$$

where the vector $Y^{(n)}$ has a Gaussian $\mathcal{N}\left(0, I_{n}\right)$ distribution. In order to investigate the large deviation properties of $\left(\mathcal{W}_{n}(f)\right)$, it is necessary to calculate the normalized cumulant generating function given, for all $t \in \mathbb{R}$, by

$$
L_{n}(t)=\frac{1}{n} \log \mathbb{E}\left[\exp \left(n t \mathcal{W}_{n}(f)\right)\right]
$$

For convenience and in all the sequel, we use of the notation that $\log t=-\infty$ if $t \leq 0$. We deduce from (3.2) and standard Gaussian calculation that for all $t \in \mathbb{R}$

$$
\begin{aligned}
L_{n}(t) & =-\frac{1}{2 n} \log \operatorname{det}\left(I_{n}-2 t T_{n}(g)^{1 / 2} T_{n}(f) T_{n}(g)^{1 / 2}\right) \\
& =-\frac{1}{2 n} \sum_{k=0}^{n} \log \left(1-2 t \lambda_{k}^{n}\right)
\end{aligned}
$$

where $\lambda_{0}^{n}, \ldots, \lambda_{n}^{n}$ are the eigenvalues of $T_{n}(g)^{1 / 2} T_{n}(f) T_{n}(g)^{1 / 2}$. For all $t \in \mathbb{R}$, let

$$
L_{f g}(t)=-\frac{1}{4 \pi} \int_{\mathbb{T}} \log (1-2 t f(x) g(x)) d x,
$$


and denote by $I_{f g}$ its Fenchel-Legendre transform

$$
I_{f g}(x)=\sup _{t \in \mathbb{R}}\left\{x t-L_{f g}(t)\right\}
$$

Furthermore, for all $x \in \mathbb{R}$, let

$$
J_{f g}(x)= \begin{cases}I_{f g}(a)+\frac{1}{2 \lambda_{\min }(f, g)}(x-a) & \text { if } x \in]-\infty, a] \\ I_{f g}(x) & \text { if } x \in] a, b[ \\ I_{f g}(b)+\frac{1}{2 \lambda_{\max }(f, g)}(x-b) & \text { if } x \in[b,+\infty[\end{cases}
$$

where $a$ and $b$ are the extended real numbers given by

$$
a=L_{f g}^{\prime}\left(\frac{1}{2 \lambda_{\min }(f, g)}\right)
$$

if $\lambda_{\min }(f, g)<0$ and $\lambda_{\min }(f, g)<\inf (f g), a=-\infty$ otherwise, while

$$
b=L_{f g}^{\prime}\left(\frac{1}{2 \lambda_{\max }(f, g)}\right)
$$

if $\lambda_{\max }(f, g)>0$ and $\lambda_{\max }(f, g)>\sup (f g), b=+\infty$ otherwise. We immediately deduce from Theorem 1 of [1] together with Corollary 2.2, that an LDP holds for $\left(\mathcal{W}_{n}(f)\right)$.

Theorem 3.1 The sequence $\left(\mathcal{W}_{n}(f)\right)$ satisfies an $L D P$ with good rate function $J_{f g}$. More precisely, for any closed set $F \subset \mathbb{R}$

$$
\limsup _{n \rightarrow \infty} \frac{1}{n} \log \mathbb{P}\left(\mathcal{W}_{n}(f) \in F\right) \leq-\inf _{x \in F} J_{f g}(x)
$$

while for any open set $G \subset \mathbb{R}$

$$
\liminf _{n \rightarrow \infty} \frac{1}{n} \log \mathbb{P}\left(\mathcal{W}_{n}(f) \in G\right) \geq-\inf _{x \in G} J_{f g}(x)
$$

Remark 3.2 Denote by $\mu$ the derivative of $L_{f g}$ at point zero

$$
\mu=\frac{1}{2 \pi} \int_{\mathbb{T}} f(x) g(x) d x
$$

Then, we have $J_{f g}(\mu)=0$ and it follows from Theorem 3.1 that for all $x>\mu$

$$
\lim _{n \rightarrow \infty} \frac{1}{n} \log \mathbb{P}\left(\mathcal{W}_{n}(f) \geq x\right)=-J_{f g}(x)
$$

whereas for all $x<\mu$

$$
\lim _{n \rightarrow \infty} \frac{1}{n} \log \mathbb{P}\left(\mathcal{W}_{n}(f) \leq x\right)=-J_{f g}(x)
$$




\section{An illustrative example}

Let $a$ and $\theta$ be two real numbers with $|\theta|<1$ and consider the two bounded continuous real functions $f$ and $g$ given by

$$
f(x)=a+\cos (x) \quad \text { and } \quad g(x)=\frac{1}{1+\theta^{2}-2 \theta \cos (x)} .
$$

The goal of this section is to study the limiting set of eigenvalues of the sequence $\left(T_{n}(f) T_{n}(g)\right)$. We clearly have $\|f\|_{\infty}=|a|+1$ and $\|g\|_{\infty}=(1-|\theta|)^{-2}$. The function $g$ is simply the spectral density of a Gaussian autoregressive process [1]. If $\theta=0, g=1$ and the product $T_{n}(f) T_{n}(g)$ reduces to $T_{n}(f)$. Consequently, $\lambda_{\max }(f, 1)=a+1$ and $\lambda_{\min }(f, 1)=a-1$. If $\theta \neq 0$, denote

$$
a_{\theta}=-\frac{(1+\theta)}{2 \theta} \quad \text { and } \quad b_{\theta}=-\frac{(1-\theta)}{2 \theta} \text {. }
$$

It is more convenient to work with the inverse of $T_{n}(g)$. As a matter of fact, $T_{n}(g)^{-1}$ is a tridiagonal matrix quite similar to $T_{n}\left(g^{-1}\right)$ except that, at the two diagonal corners of $T_{n}\left(g^{-1}\right)$, the coefficient $1+\theta^{2}$ is replaced by 1

$$
T_{n}(g)^{-1}=\left(\begin{array}{cccc}
1 & -\theta & 0 & \ldots \\
-\theta & 1+\theta^{2} & -\theta & \ldots \\
\ldots & \ldots & \ldots & \ldots \\
\cdots & -\theta & 1+\theta^{2} & -\theta \\
\cdots & 0 & -\theta & 1
\end{array}\right) .
$$

It is not hard to see that $\operatorname{det}\left(T_{n}(g)^{-1}\right)=1-\theta^{2}$. In order to find the eigenvalues $\lambda$ of the product $T_{n}(f) T_{n}(g)$, it is equivalent to calculate the zeros of its characteristic polynomial which correspond also to the zeros of $\operatorname{det}\left(M_{n}(t)\right)$ where

$$
M_{n}(t)=t T_{n}(f)-T_{n}(g)^{-1}
$$

with $t=1 / \lambda$. As $T_{n}(f)$ and $T_{n}(g)^{-1}$ are both tridiagonal matrices, we can easily compute $\operatorname{det}\left(M_{n}(t)\right)$. Via the same lines than in Lemma 11 of [1], we find that for $n$ large enough, $M_{n}(t)$ is negative definite only on the domain $\mathcal{D}=\mathcal{D}_{1} \cup \mathcal{D}_{2}$ with

$$
\begin{aligned}
& \mathcal{D}_{1}=\left\{-2 \theta^{2}<p \leq-\theta^{2} \text { and } q^{2}<-4 \theta^{2}\left(p+\theta^{2}\right)\right\}, \\
& \mathcal{D}_{2}=\left\{p<-2 \theta^{2} \text { and } p<-|q|\right\},
\end{aligned}
$$

where $p=a t-\left(1+\theta^{2}\right)$ and $q=t+2 \theta$. In term of the variable $\lambda$, the inverses of the boundaries of $\mathcal{D}$ give the extrema of $\sigma(T(f) T(g))$ that is $\lambda_{\max }(f, g)$ and $\lambda_{\min }(f, g)$. After some tedious but straightforward calculations, we obtain three inverses of the boundaries

$$
\frac{a-1}{(1+\theta)^{2}}, \quad \frac{a+1}{(1-\theta)^{2}}, \quad-\frac{1}{4 \theta(1+a \theta)} .
$$

Two of them coincide with $\inf (f g)$ and $\sup (f g)$. It only depends on the location of $a$ with respect to $-\left(1+\theta^{2}\right) /(2 \theta)$. The last one can be $\lambda_{\max }(f, g)>\sup (f g)$ or $\lambda_{\min }(f, g)<\inf (f g)$. It only depends on the sign of $\theta$ as well as on the location of $a$ with respect to the interval $\left[a_{\theta}, b_{\theta}\right]$. More precisely, if $\theta>0$ then $\lambda_{\max }(f, g)=\sup (f g)$ while

$$
\lambda_{\min }(f, g)=\frac{1}{-4 \theta(1+a \theta)}<\inf (f g)=\min \left(\frac{a-1}{(1+\theta)^{2}}, \frac{a+1}{(1-\theta)^{2}}\right)
$$

if $a \in] a_{\theta}, b_{\theta}\left[\right.$ and $\lambda_{\min }(f, g)=\inf (f g)$ otherwise. Moreover, if $\theta<0$ then $\lambda_{\min }(f, g)=\inf (f g)$ while

$$
\lambda_{\max }(f, g)=\frac{1}{-4 \theta(1+a \theta)}>\sup (f g)=\max \left(\frac{a-1}{(1+\theta)^{2}}, \frac{a+1}{(1-\theta)^{2}}\right)
$$

if $a \in] a_{\theta}, b_{\theta}\left[\right.$ and $\lambda_{\max }(f, g)=\sup (f g)$ otherwise. 


\section{Toeplitz operators and functional calculus}

We will prove the following result which implies Corollary 2.2 for continuous fonctions.

Theorem 5.1 Let $f$ and $g$ be two bounded continuous real functions with $g \geq 0$. For $\lambda \in \mathbb{R}$, the following properties are equivalent:

i) $\lambda \in \sigma(T(f) T(g))$,

ii) $\lambda$ is the limit of a sequence $\left(\lambda_{n}\right)$ where $\lambda_{n} \in \sigma\left(T_{n}(f) T_{n}(g)\right)$,

iii) $\lambda$ is the limit of a subsequence $\left(\lambda_{n_{k}}\right)$ where $\lambda_{n_{k}} \in \sigma\left(T_{n_{k}}(f) T_{n_{k}}(g)\right)$.

First, let us interpret the projection operators $P_{n}$ and $P$ as spectral projectors of the derivation operator and introduce the main ingredients of the proofs.

\subsection{A functional point of view}

We consider the Toeplitz operators $T(f)$ and $T_{n}(f)$ as the cut-off, in frequencies, of the operator of multiplication by $f$. To be more precise, let us introduce the Fourier transform, $\mathcal{F}: L^{2}(\mathbb{T}) \rightarrow \ell^{2}(\mathbb{Z})$, defined by

$$
(\mathcal{F} u)_{k}=\widehat{u}_{k}=\frac{1}{2 \pi} \int_{-\pi}^{\pi} u(x) e^{-i k x} d x .
$$

The operator $\mathcal{F}$ is an isomorphism. We denote by $\mathcal{F}^{-1}$ its inverse, and we introduce the projections $\widehat{P}$ and $\widehat{P}_{n}$ as

$$
\begin{aligned}
\widehat{P}: \widehat{u} & \in \ell^{2}(\mathbb{Z}) \longmapsto\left(\ldots, 0,0, \widehat{u}_{0}, \widehat{u}_{1}, \ldots\right) \in \ell^{2}(\mathbb{Z}) \\
\widehat{P}_{n}: \widehat{u} & \in \ell^{2}(\mathbb{Z}) \longmapsto\left(\ldots, 0,0, \widehat{u}_{0}, \widehat{u}_{1}, \ldots, \widehat{u}_{n}, 0,0, \ldots\right) \in \ell^{2}(\mathbb{Z}) .
\end{aligned}
$$

On the other hand, if we identify $f \in L^{\infty}(\mathbb{T})$ with $L(f)$, the bounded operator defined on $L^{2}(\mathbb{T})$ by

$$
u \in L^{2}(\mathbb{T}) \longmapsto f u \in L^{2}(\mathbb{T}),
$$

we have

$$
T(f)=P f P \quad \text { and } \quad T_{n}(f)=P_{n} f P_{n},
$$

with $P=\mathcal{F}^{-1} \widehat{P} \mathcal{F}$ and $P_{n}=\mathcal{F}^{-1} \widehat{P}_{n} \mathcal{F}$. In the following, we will systematically identify $f$ with the operator $L(f)$. Since

$$
\frac{1}{i} \frac{d}{d x}\left(e^{i k x}\right)=k e^{i k x}
$$

the derivation operator $D$ defined on

$$
H^{1}(\mathbb{T})=\left\{u \in L^{2}(\mathbb{T}) ; \frac{d}{d x} u \in L^{2}(\mathbb{T})\right\}=\left\{u \in L^{2}(\mathbb{T}) ;\left(k \hat{u}_{k}\right)_{k} \in \ell^{2}(\mathbb{Z})\right\}
$$

by

$$
D: u \in H^{1}(\mathbb{T}) \longmapsto \frac{1}{i} \frac{d}{d x} u \in L^{2}(\mathbb{T})
$$

is self-adjoint on $L^{2}(\mathbb{T})$ and $\mathcal{F} D \mathcal{F}^{-1}$ is the diagonal operator $\left(k \delta_{k, j}\right)_{k, j \in \mathbb{Z}}$. For any bounded Borel function $\varphi$, the bounded operator $\varphi(D)$ is defined with the help of the functional calculus for selfadjoint operators. It satisfies

$$
\varphi(D)=\mathcal{F}^{-1} M(\varphi) \mathcal{F},
$$


where $M(\varphi)$ is the operator

$$
\widehat{u} \in \ell^{2}(\mathbb{Z}) \longmapsto\left(\ldots, \varphi(k) \widehat{u}_{k}, \ldots\right) \in \ell^{2}(\mathbb{Z}) .
$$

In particular, if $\mathbf{1}_{I}$ denotes the indicator function of the interval $I$, we have

$$
\mathbf{1}_{[0,+\infty[}(D)=P \quad \text { and } \quad \mathbf{1}_{[0, n]}(D)=\mathbf{1}_{[0,1]}\left(n^{-1} D\right)=P_{n} .
$$

Moreover, note that if $\operatorname{supp}(\varphi) \subset[a, b]$, we have the trivial properties

$$
\mathbf{1}_{[a, b]}(D) \varphi(D)=\varphi(D) \quad \text { and } \quad \mathbf{1}_{[a, b]}(D) e^{i k x}=e^{i k x} \mathbf{1}_{[a-k, b-k]}(D) .
$$

In the rest of the paper, a function is a $o_{a \rightarrow b}^{c}(1)$ if, for each $c$ fixed, the function goes to 0 as $a$ tends to $b$. In the same way, a function is a $\mathcal{O}^{c}(1)$ if, for each $c$ fixed, the function is a $\mathcal{O}(1)$.

\subsection{A commutator estimate}

In this subsection, we recall a standard result of the functional analysis. For $\rho \in \mathbb{R}$, we denote by $S^{\rho}(\mathbb{R})$ the class of functions $\varphi$ in $C^{\infty}(\mathbb{R})$ such that

$$
\left|\partial_{s}^{k} \varphi(s)\right| \leq C_{k}\langle s\rangle^{\rho-k}
$$

for $k \geq 0$. Here $\langle x\rangle=\left(1+|x|^{2}\right)^{1 / 2}$.

Lemma 5.2 (Lemma C.3.2 of [8]) Let $A, B$ be self-adjoint operators on a Hilbert space with $B$ and $[A, B]$ bounded. If $\varphi \in S^{\rho}(\mathbb{R})$ with $\rho<1$, then

$$
\|[\varphi(A), B]\| \leq C_{\varphi}\|[A, B]\| .
$$

Here, $[A, B]=A B-B A$ denotes the commutator. The constant $C_{\varphi}$ only depends on $\varphi$.

Applying this lemma, we immediately obtain

Lemma 5.3 Let $f \in C^{0}(\mathbb{T})$ and $\varphi \in S^{\rho}(\mathbb{R})$ with $\rho \leq 0$. Then

$$
[\varphi(\varepsilon D), f]=o_{\varepsilon \rightarrow 0}(1)
$$

Proof By Weierstrass's theorem, there exist $f_{k} \in C^{1}(\mathbb{T})$ satisfying $f_{k} \rightarrow f$ in $L^{\infty}(\mathbb{T})$. Then, viewed as operators, we have $f_{k} \rightarrow f$. Remark that $\left[\varepsilon D, f_{k}\right]=-\varepsilon i f_{k}^{\prime}$. From Lemma 5.2 , we obtain

$$
\left\|\left[\varphi(\varepsilon D), f_{k}\right]\right\| \leq \varepsilon C_{\varphi}\left\|f_{k}^{\prime}\right\|_{\infty} .
$$

Then, using the assumption that $\varphi$ is bounded,

$$
\begin{aligned}
{[\varphi(\varepsilon D), f] } & =\left[\varphi(\varepsilon D), f_{k}\right]+o_{k \rightarrow \infty}(1)=\mathcal{O}^{k}(\varepsilon)+o_{k \rightarrow \infty}(1) \\
& =o_{\varepsilon \rightarrow 0}(1),
\end{aligned}
$$

since $[\varphi(\varepsilon D), f]$ does not depend on $k$. 
5.3 Essential spectrum of the product of Toeplitz operators

Here, we recall, in our setting, a consequence of a theorem of Coburn [5] concerning the essential spectrum of the product of Toeplitz operators. This result has been extended by Douglas to a more general framework (see [12, Theorem 4.5.10]). We shall give in section 7 an alternative proof of the following theorem, more related to our approach.

Theorem 5.4 (Coburn) Let $f$ and $g$ be two bounded continuous real functions with $g \geq 0$. The bounded self-adjoint operator $T(g)^{1 / 2} T(f) T(g)^{1 / 2}$ satisfies on $\operatorname{Im} P$

$$
\sigma_{\text {ess }}\left(T(g)^{1 / 2} T(f) T(g)^{1 / 2}\right)=[\inf (f g), \sup (f g)] .
$$

Here, $\sigma_{\mathrm{ess}}(A)$ denotes the essential spectrum of $A$.

In Theorem 5.4, the operator $T(g)^{1 / 2} T(f) T(g)^{1 / 2}$ is viewed as an operator on $\operatorname{Im} P$. On $L^{2}(\mathbb{T})$, this operator is a block diagonal operator with respect to the orthogonal sum $L^{2}=\operatorname{Im} P \oplus^{\perp} \operatorname{Im}(1-P)$ and is equal to 0 on $\operatorname{Im}(1-P)$. In particular, we have

Remark 5.5 If the operator $T(g)^{1 / 2} T(f) T(g)^{1 / 2}$ is viewed on $L^{2}(\mathbb{T})$, we have

$$
\sigma_{\text {ess }}\left(T(g)^{1 / 2} T(f) T(g)^{1 / 2}\right)=[\inf (f g), \sup (f g)] \cup\{0\} .
$$

\section{Proof of Theorem 5.1}

The goal of this section is to prove Theorem 5.1. First of all, one can observe that part ii) clearly implies iii). In the next subsection, we first show that i) implies ii).

6.1 The implication i) gives ii)

Lemma 6.1 Let $f$ and $g$ be two bounded piecewise continuous real functions with $g \geq 0$. Then,

$$
T_{n}(g)^{1 / 2} T_{n}(f) T_{n}(g)^{1 / 2} \longrightarrow T(g)^{1 / 2} T(f) T(g)^{1 / 2}
$$

strongly on $L^{2}(\mathbb{T})$. If $\lambda$ belongs to the spectrum of $T(f) T(g)$ on $\operatorname{Im} P$, then there exists an eigenvalue $\lambda_{n}$ of $T_{n}(f) T_{n}(g)$ on $\operatorname{Im} P_{n}$ such that $\lambda_{n} \rightarrow \lambda$.

Proof Since $P_{n} \longrightarrow P$, it follows from Lemma III.3.8 of [11] that for all $f \in L^{\infty}(\mathbb{T}), T_{n}(f) \longrightarrow T(f)$. In particular, from Problem VI.14 of [13] (see also Theorem VI.9 of [13]), $T_{n}(g)^{1 / 2} \longrightarrow T(g)^{1 / 2}$. Consequently, we deduce from Lemma III.3.8 of [11] that

$$
T_{n}(g)^{1 / 2} T_{n}(f) T_{n}(g)^{1 / 2} \longrightarrow T(g)^{1 / 2} T(f) T(g)^{1 / 2},
$$

on $L^{2}(\mathbb{T})$. In particular, we obtain on $\operatorname{Im} P$

$$
T_{n}(g)^{1 / 2} T_{n}(f) T_{n}(g)^{1 / 2}+M\left(P-P_{n}\right) \longrightarrow T(g)^{1 / 2} T(f) T(g)^{1 / 2},
$$

for all $M \in \mathbb{R}$. We choose $\mu=\|f\|_{\infty}\|g\|_{\infty}$ and $M=\mu+1$. Therefore, it follows from Corollary VIII.1.6 together with Theorem VIII.1.14 of [11] that, for each $\lambda$ belonging to the spectrum $\sigma(T(f) T(g))=$ $\sigma\left(T(g)^{1 / 2} T(f) T(g)^{1 / 2}\right)$ on $\operatorname{Im} P$, there exists an eigenvalue $\lambda_{n}$ of the matrix

$$
T_{n}(g)^{1 / 2} T_{n}(f) T_{n}(g)^{1 / 2}+M\left(P-P_{n}\right),
$$


on $\operatorname{Im} P$ such that $\lambda_{n} \rightarrow \lambda$. As $\left\|T(g)^{1 / 2} T(f) T(g)^{1 / 2}\right\| \leq \mu$, we necessarily have $\lambda \in[-\mu, \mu]$ and then $M \geq|\lambda|+1$. In particular, for $n$ large enough, $M>\left|\lambda_{n}\right|+1 / 2$. Therefore, $\lambda_{n}$ is an eigenvalue of $T_{n}(g)^{1 / 2} T_{n}(f) T_{n}(g)^{1 / 2}$ on $\operatorname{Im} P_{n}$ because

$$
T_{n}(g)^{1 / 2} T_{n}(f) T_{n}(g)^{1 / 2}+M\left(P-P_{n}\right)=T_{n}(g)^{1 / 2} T_{n}(f) T_{n}(g)^{1 / 2} \oplus^{\perp} M\left(P-P_{n}\right),
$$

is a block diagonal operator with respect to the orthogonal $\operatorname{sum} \operatorname{Im} P=\operatorname{Im} P_{n} \oplus^{\perp} \operatorname{Im}\left(P-P_{n}\right)$.

6.2 The implication iii) gives $i$ )

Let $\lambda_{N}$ be a sequence of eigenvalues of $T_{N}(f) T_{N}(g)$ such that $\lambda_{N} \rightarrow \lambda \in \mathbb{R}$. Here $N$ is a subsequence of $\mathbb{N}$ and we have to show that $\lambda$ is in the spectrum of $T(f) T(g)$. From Theorem 5.4 , we know that $[\inf (f g), \sup (f g)]$ is always inside the spectrum of $T(f) T(g)$. Thus, we can assume that

$$
\lambda \notin[\inf (f g), \sup (f g)] .
$$

By Weierstrass's theorem, there exists a sequence of functions $\left(f_{k}\right) \in C^{\infty}(\mathbb{T})$ such that $f_{k} \rightarrow f$ in $L^{\infty}(\mathbb{T})$ and supp $\widehat{f_{k}} \subset[-k, k]$. We also consider $\left(g_{k}\right)$ a sequence corresponding to $g$ with the same properties mutatis mutandis. In particular, for all $n \in \mathbb{N}$,

$$
T_{n}(f)=T_{n}\left(f_{k}\right)+o_{k \rightarrow \infty}(1) \quad \text { and } \quad T(f)=T\left(f_{k}\right)+o_{k \rightarrow \infty}(1) .
$$

Recall that, by definition, a $o_{k \rightarrow \infty}(1)$ is uniform with respect to $n$.

Finally, let $u_{N} \in \operatorname{Im} P_{N}$ be an eigenvector of $T_{N}(f) T_{N}(g)$ associated with $\lambda_{N}$ and satisfying $\left\|u_{N}\right\|=1$. From (6.3),

$$
\begin{gathered}
T_{N}(f) T_{N}(g) u_{N}=\lambda_{N} u_{N}=\lambda u_{N}+o_{N \rightarrow \infty}(1) \\
T_{N}\left(f_{k}\right) T_{N}\left(g_{k}\right) u_{N}=\lambda u_{N}+o_{N \rightarrow \infty}(1)+o_{k \rightarrow \infty}(1) .
\end{gathered}
$$

In the following, we denote $D_{n}=n^{-1} D$.

\subsubsection{Localization of the eigenvectors}

Lemma 6.2 Let $\varphi \in C_{0}^{\infty}(] 0,1[)$. Then, in $L^{2}(\mathbb{T})$ norm,

$$
\varphi\left(D_{N}\right) u_{N}=o_{N \rightarrow \infty}(1) .
$$

Proof From Lemma 5.3, we have

$$
\begin{aligned}
\varphi\left(D_{N}\right) T_{N}(f) & =\varphi\left(D_{N}\right) \mathbf{1}_{[0,1]}\left(D_{N}\right) f \mathbf{1}_{[0,1]}\left(D_{N}\right)=\varphi\left(D_{N}\right) f \mathbf{1}_{[0,1]}\left(D_{N}\right) \\
& =f \varphi\left(D_{N}\right) \mathbf{1}_{[0,1]}\left(D_{N}\right)+o_{N \rightarrow \infty}(1) \\
& =f \varphi\left(D_{N}\right)+o_{N \rightarrow \infty}(1) .
\end{aligned}
$$

Applying two times this estimate, we obtain

$$
\begin{aligned}
\varphi\left(D_{N}\right) T_{N}(f) T_{N}(g) u_{N} & =f \varphi\left(D_{N}\right) T_{N}(g) u_{N}+o_{N \rightarrow \infty}(1) \\
& =f g \varphi\left(D_{N}\right) u_{N}+o_{N \rightarrow \infty}(1) .
\end{aligned}
$$

Then, (6.4) gives

$$
(f g-\lambda) \varphi\left(D_{N}\right) u_{N}=o_{N \rightarrow \infty}(1) .
$$

Since $\lambda \notin[\inf (f g), \sup (f g)]$, the function $(f g-\lambda)^{-1}$ belongs to $L^{\infty}(\mathbb{T})$ and the lemma follows from the last equation. 
Now, we take $\varphi \in C_{0}^{\infty}(] 0,1[,[0,1])$ such that $\varphi=1$ near $[\varepsilon, 1-\varepsilon]$ for $\varepsilon>0$ small enough (we choose $\varepsilon=1 / 8)$. Let $\varphi^{-} \in C_{0}^{\infty}([-\varepsilon, 2 \varepsilon],[0,1])$ and $\varphi^{+} \in C_{0}^{\infty}([1-2 \varepsilon, 1+\varepsilon],[0,1])$ be two functions such that

$$
\varphi^{-}+\varphi+\varphi^{+}=1
$$

in the neighborhood of $[0,1]$. Set

$$
u_{N}^{ \pm}=\varphi^{ \pm}\left(D_{N}\right) u_{N}=\varphi^{ \pm}\left(D_{N}\right) \mathbf{1}_{[0,1]}\left(D_{N}\right) u_{N}
$$

As $\left\|u_{N}\right\|=1$, it follows from Lemma 6.2 that

$$
\left\|u_{N}^{-}+u_{N}^{+}\right\|=1+o_{N \rightarrow \infty}(1) .
$$

In particular, we can assume, up to the extraction of a subsequence, that

$$
\forall N \quad\left\|u_{N}^{-}\right\| \geq 1 / 3 \quad \text { or } \quad \forall N \quad\left\|u_{N}^{+}\right\| \geq 1 / 3 .
$$

In the next section, we will suppose that

$$
\left\|u_{N}^{-}\right\| \geq 1 / 3
$$

The case $\left\|u_{N}^{+}\right\| \geq 1 / 3$ follows essentially the same lines and is treated in Section 6.2.3. But before, we show that $u_{N}^{-}$and $u_{N}^{+}$are both quasimodes of $T_{N}(f) T_{N}(g)$ (this means that they are eigenvectors modulo a small term).

Lemma 6.3 We have

$$
T_{N}\left(f_{k}\right) T_{N}\left(g_{k}\right) u_{N}^{ \pm}=\lambda u_{N}^{ \pm}+o_{k \rightarrow \infty}(1)+o_{N \rightarrow \infty}^{k}(1)
$$

Proof As in (6.6), using Lemma 5.3, we get

$$
\begin{aligned}
T_{N}\left(f_{k}\right) T_{N}\left(g_{k}\right) u_{N}^{ \pm} & =\mathbf{1}_{[0,1]}\left(D_{N}\right) f_{k} \mathbf{1}_{[0,1]}\left(D_{N}\right) g_{k} \mathbf{1}_{[0,1]}\left(D_{N}\right) \varphi^{ \pm}\left(D_{N}\right) u_{N} \\
& =\mathbf{1}_{[0,1]}\left(D_{N}\right) f_{k} \mathbf{1}_{[0,1]}\left(D_{N}\right) g_{k} \varphi^{ \pm}\left(D_{N}\right) \mathbf{1}_{[0,1]}\left(D_{N}\right) u_{N} \\
& =\mathbf{1}_{[0,1]}\left(D_{N}\right) f_{k} \mathbf{1}_{[0,1]}\left(D_{N}\right) \varphi^{ \pm}\left(D_{N}\right) g_{k} \mathbf{1}_{[0,1]}\left(D_{N}\right) u_{N}+o_{N \rightarrow \infty}^{k}(1) \\
& =\mathbf{1}_{[0,1]}\left(D_{N}\right) \varphi^{ \pm}\left(D_{N}\right) f_{k} \mathbf{1}_{[0,1]}\left(D_{N}\right) g_{k} \mathbf{1}_{[0,1]}\left(D_{N}\right) u_{N}+o_{N \rightarrow \infty}^{k}(1) \\
& =\varphi^{ \pm}\left(D_{N}\right) T_{N}\left(f_{k}\right) T_{N}\left(g_{k}\right) u_{N}+o_{N \rightarrow \infty}^{k}(1) .
\end{aligned}
$$

The lemma follows from (6.5), (6.7) and the last identity.

\subsubsection{Concentration near the low frequencies}

Here, we assume (6.9) and we prove that $u_{N}^{-}$, viewed as an element of $\operatorname{Im} P$, is a quasimode of $T\left(f_{k}\right) T\left(g_{k}\right)$.

Lemma 6.4 For $4 k \leq N$, we have

$$
T\left(f_{k}\right) T\left(g_{k}\right) u_{N}^{-}=T_{N}\left(f_{k}\right) T_{N}\left(g_{k}\right) u_{N}^{-}
$$

Remark 6.5 In fact, for $4 k \leq N \leq n$, we have

$$
T_{n}\left(f_{k}\right) T_{n}\left(g_{k}\right) u_{N}^{-}=T_{N}\left(f_{k}\right) T_{N}\left(g_{k}\right) u_{N}^{-} .
$$


Proof Recall that, if $u, v$ are two functions of $L^{2}(\mathbb{T})$ such that $\operatorname{supp} \widehat{u} \subset[a, b]$ and $\operatorname{supp} \widehat{v} \subset[c, d]$, then $\operatorname{supp} \widehat{u v} \subset[a+c, b+d]$. By definition,

$$
T\left(f_{k}\right) T\left(g_{k}\right) u_{N}^{-}=P f_{k} P g_{k} P \varphi^{-}\left(D_{N}\right) u_{N}=P f_{k} P g_{k} P_{N} \varphi^{-}\left(D_{N}\right) u_{N} .
$$

Since supp $\widehat{g_{k}} \subset[-k, k]$ and $\operatorname{supp} \mathcal{F}\left(P_{N} \varphi^{-}\left(D_{N}\right) u_{N}\right) \subset[0, N / 4]$, the Fourier transform of the function $g_{k} P_{N} \varphi^{-}\left(D_{N}\right) u_{N}$ is supported inside $[-k, N / 4+k] \subset[-k, N]$. In particular,

$$
P g_{k} P_{N} \varphi^{-}\left(D_{N}\right) u_{N}=P_{N} g_{k} P_{N} \varphi^{-}\left(D_{N}\right) u_{N}
$$

and the Fourier transform of this function is supported inside $[0, N / 4+k]$. As before, the Fourier transform of

$$
f_{k} P_{N} g_{k} P_{N} \varphi^{-}\left(D_{N}\right) u_{N}
$$

is supported inside $[-k, N / 4+2 k] \subset[-k, N]$. Then

$$
P f_{k} P_{N} g_{k} P_{N} \varphi^{-}\left(D_{N}\right) u_{N}=P_{N} f_{k} P_{N} g_{k} P_{N} \varphi^{-}\left(D_{N}\right) u_{N}
$$

The lemma follows from (6.11), (6.12) and (6.13).

From (6.3), Lemma 6.3 and Lemma 6.4, we get

$$
T(f) T(g) u_{N}^{-}=\lambda u_{N}^{-}+o_{k \rightarrow \infty}(1)+o_{N \rightarrow \infty}^{k}(1),
$$

for $4 k \leq N$. If $\lambda \notin \sigma(T(f) T(g))$, the operator $T(f) T(g)-\lambda$ is invertible and then

$$
u_{N}^{-}=o_{k \rightarrow \infty}(1)+o_{N \rightarrow \infty}^{k}(1) \text {. }
$$

From (6.9), we obtain $1 / 3 \leq o_{k \rightarrow \infty}(1)+o_{N \rightarrow \infty}^{k}(1)$. Taking $k$ large enough and then $N$ large enough, it is clear that this is impossible. Thus,

$$
\lambda \in \sigma(T(f) T(g)),
$$

which implies Theorem 5.1 under the assumption (6.9).

\subsubsection{Concentration near the high frequencies}

We replace the assumption $(6.9)$ by $\left\|u_{N}^{+}\right\| \geq 1 / 3$. Let $J$ be the isometry $f \mapsto \tilde{f}$ in $L^{2}(\mathbb{T})$. One can observe that $J(u v)=J(u) J(v)$. Using the notation $P_{[a, b]}=\mathbf{1}_{[a, b]}(D)$, we have $P_{[a, b]} J=J P_{[-b,-a]}$ and $P_{[a, b]} e^{i c x}=e^{i c x} P_{[a-c, b-c]}$. Combining these identities with Lemma 6.3, we get

$$
\begin{aligned}
T_{N}\left(J f_{k}\right) T_{N}\left(J g_{k}\right) e^{i N x}\left(J u_{N}^{+}\right) & =P_{[0, N]}\left(J f_{k}\right) P_{[0, N]}\left(J g_{k}\right) P_{[0, N]} e^{i N x}\left(J u_{N}^{+}\right) \\
& =e^{i N x} P_{[-N, 0]}\left(J f_{k}\right) P_{[-N, 0]}\left(J g_{k}\right) P_{[-N, 0]}\left(J u_{N}^{+}\right) \\
& =e^{i N x} J P_{[0, N]} f_{k} P_{[0, N]} g_{k} P_{[0, N]} u_{N}^{+} \\
& =\lambda e^{i N x}\left(J u_{N}^{+}\right)+o_{k \rightarrow \infty}(1)+o_{N \rightarrow \infty}^{k}(1) .
\end{aligned}
$$

In particular, $\widetilde{u}_{N}^{-}=e^{i N x}\left(J u_{N}^{+}\right)$satisfies $\left\|\widetilde{u}_{N}^{-}\right\| \geq 1 / 3$

$$
T_{N}\left(J f_{k}\right) T_{N}\left(J g_{k}\right) \widetilde{u}_{N}^{-}=\lambda \widetilde{u}_{N}^{-}+o_{k \rightarrow \infty}(1)+o_{N \rightarrow \infty}^{k}(1)
$$

and the support of the Fourier transform of $\widetilde{u}_{N}^{-}$is inside $[0, N / 4]$. Hence, we can apply the method developed in the case $\left\|u_{N}^{-}\right\| \geq 1 / 3$. The unique difference is that $f, g$ are replaced by $\tilde{f}, \widetilde{g}$. Then, we obtain

$$
\lambda \in \sigma(T(\widetilde{f}) T(\widetilde{g})) .
$$

Theorem 5.1 follows from the following lemma and $\sigma(T(f) T(g))=\sigma(T(g) T(f)$ ) (the spectrum of $T(f) T(g)$ is real and $\left.(T(f) T(g)-z)^{*}=T(g) T(f)-\bar{z}\right)$. 
Lemma 6.6 Let $f, g \in L^{\infty}(\mathbb{T})$. Then

$$
\sigma(T(\widetilde{f}) T(\widetilde{g}))=\sigma(T(g) T(f)) .
$$

Proof For $A$ a bounded linear operator on $L^{2}$, we define $A^{t}$ by

$$
\left(A^{t} u, v\right)=(u, \overline{A \bar{v}}),
$$

for all $u, v \in L^{2}$. Simple calculi give $f^{t}=f, P_{[a, b]}^{t}=P_{[-b,-a]},(A B)^{t}=B^{t} A^{t}$ and then

$$
T(f)^{t}=\left(P_{[0,+\infty[} f P_{[0,+\infty[}\right)^{t}=P_{-\infty, 0]} f P_{-\infty, 0]} .
$$

By the same way, since $J=J^{*}=J^{-1}$,

$$
J P_{-\infty, 0]} f P_{-\infty, 0]} J=P_{[0,+\infty[} \tilde{f} P_{[0,+\infty[}=T(\widetilde{f}) .
$$

Combining these identities concerning ${ }^{t}$ and $J$, we get

$$
\begin{aligned}
J(T(\widetilde{f}) T(\widetilde{g}))^{t} J^{-1} & =J\left(P_{]-\infty, 0]} \widetilde{g} P_{-\infty, 0]}\right)\left(P_{]-\infty, 0]} \widetilde{f} P_{-\infty, 0]}\right) J \\
& =T(g) T(f) .
\end{aligned}
$$

Since $J A^{t} J-z=J(A-z)^{t} J, A$ and $J A^{t} J$ have the same spectrum and the lemma follows.

\section{Proof of Theorem 5.4}

We give here an alternative proof of Coburn's theorem. Let $\psi \in C^{\infty}(\mathbb{R})$ satisfying $\psi=1$ near $[2,+\infty[$ and $\psi=0$ near $]-\infty, 1]$. For $\varepsilon>0$, we have on $\operatorname{Im} P$

$$
\begin{aligned}
T(g)^{1 / 2} T(f) T(g)^{1 / 2} & =T(g)^{1 / 2} \psi(\varepsilon D) T(f) \psi(\varepsilon D) T(g)^{1 / 2}+\widetilde{R}_{\varepsilon} \\
& =T(g)^{1 / 2} \psi(\varepsilon D) f \psi(\varepsilon D) T(g)^{1 / 2}+\widetilde{R}_{\varepsilon},
\end{aligned}
$$

where

$$
\widetilde{R}_{\varepsilon}=T(g)^{1 / 2}(1-\psi(\varepsilon D)) T(f) \psi(\varepsilon D) T(g)^{1 / 2}+T(g)^{1 / 2} T(f)(1-\psi(\varepsilon D)) T(g)^{1 / 2},
$$

is a self-adjoint operator of finite rank. Recall that if $A \geq 0$ is a bounded operator with $\|A\| \leq 1$, then

$$
A^{1 / 2}=\sum_{j=0}^{+\infty} c_{j}(1-A)^{j},
$$

where $\|1-A\| \leq 1$ and $\sum_{j \geq 0}\left|c_{j}\right| \leq 2<+\infty$. On the other hand, Lemma 5.3 implies

$$
\begin{aligned}
T(g) \psi(\varepsilon D) & =P g P \psi(\varepsilon D)=P g \psi(\varepsilon D)=P \psi(\varepsilon D) g+o_{\varepsilon \rightarrow 0}(1) \\
& =\psi(\varepsilon D) g+o_{\varepsilon \rightarrow 0}(1),
\end{aligned}
$$

Then, for a fixed $\delta>0$ such that $\|T(g)\| \leq\|g\|_{\infty}<\delta^{-1}$, we have

$$
\begin{aligned}
T(g)^{1 / 2} \psi(\varepsilon D) & =\delta^{-1 / 2} T(\delta g)^{1 / 2} \psi(\varepsilon D) \\
& =\delta^{-1 / 2} \sum_{j=0}^{+\infty} c_{j}(1-T(\delta g))^{j} \psi(\varepsilon D) \\
& =\delta^{-1 / 2} \sum_{j=0}^{J} c_{j}(1-T(\delta g))^{j} \psi(\varepsilon D)+o_{J \rightarrow \infty}(1) \\
& =\delta^{-1 / 2} \psi(\varepsilon D) \sum_{j=0}^{J} c_{j}(1-\delta g)^{j}+o_{J \rightarrow \infty}(1)+o_{\varepsilon \rightarrow 0}^{J}(1) \\
& =\delta^{-1 / 2} \psi(\varepsilon D)(\delta g)^{1 / 2}+o_{J \rightarrow \infty}(1)+o_{\varepsilon \rightarrow 0}^{J}(1) \\
& =\psi(\varepsilon D) g^{1 / 2}+o_{\varepsilon \rightarrow 0}(1),
\end{aligned}
$$


since these quantities do not depend on $J$. Using this identity and its adjoint, (7.1) becomes

$$
\begin{aligned}
T(g)^{1 / 2} T(f) T(g)^{1 / 2} & =\psi(\varepsilon D) f g \psi(\varepsilon D)+\widetilde{R}_{\varepsilon}+o_{\varepsilon \rightarrow 0}(1) \\
& =T(f g)+R_{\varepsilon}+e_{\varepsilon}
\end{aligned}
$$

where $e_{\varepsilon}=o_{\varepsilon \rightarrow 0}(1)$ and

$$
R_{\varepsilon}=\widetilde{R}_{\varepsilon}+(\psi(\varepsilon D)-1) T(f g) \psi(\varepsilon D)+T(f g)(\psi(\varepsilon D)-1),
$$

is a self-adjoint operator of finite rank. In particular, $e_{\varepsilon}$ is a self-adjoint operator. Since, on $\operatorname{Im} P$

$$
\inf (f g) \leq T(f g) \leq \sup (f g)
$$

we get $\sigma\left(T(f g)+e_{\varepsilon}\right) \subset\left[\inf (f g)-o_{\varepsilon \rightarrow 0}(1), \sup (f g)+o_{\varepsilon \rightarrow 0}(1)\right]$. As $R_{\varepsilon}$ is of finite rank, we obtain, from Weyl's theorem [13, Theorem S.13],

$$
\sigma_{\mathrm{ess}}\left(T(g)^{1 / 2} T(f) T(g)^{1 / 2}\right)=\sigma_{\mathrm{ess}}\left(T(f g)+e_{\varepsilon}\right) \subset\left[\inf (f g)-o_{\varepsilon \rightarrow 0}(1), \sup (f g)+o_{\varepsilon \rightarrow 0}(1)\right]
$$

As the essential spectrum of $T(g)^{1 / 2} T(f) T(g)^{1 / 2}$ does not depend on $\varepsilon$, we get

$$
\sigma_{\mathrm{ess}}\left(T(g)^{1 / 2} T(f) T(g)^{1 / 2}\right) \subset[\inf (f g), \sup (f g)],
$$

which is the first inclusion of Coburn's theorem.

Now, let $\varphi \in C^{\infty}([-1,1],[0,1])$ with $\|\varphi\|_{L^{2}}=1$. For $x_{0} \in \mathbb{T}$ and $\alpha, \beta \in \mathbb{N}$, we set

$$
u=\alpha^{1 / 2} \varphi\left(\alpha\left(x-x_{0}\right)\right) e^{i \beta x} \quad \text { and } \quad v=P u \in \operatorname{Im} P
$$

which satisfies $\|u\|=1$. We have

$$
\begin{aligned}
(1-P) u & =\alpha^{1 / 2} \mathbf{1}_{]-\infty, 0]}(D) e^{i \beta x} \varphi\left(\alpha\left(x-x_{0}\right)\right) \\
& =\alpha^{1 / 2} e^{i \beta x} \mathbf{1}_{]-\infty,-\beta]}(D) \varphi\left(\alpha\left(x-x_{0}\right)\right) \\
& =\alpha^{1 / 2} e^{i \beta x} \mathbf{1}_{]-\infty,-\beta]}(D)(D+i)^{-M}(D+i)^{M} \varphi\left(\alpha\left(x-x_{0}\right)\right) \\
& =\mathcal{O}\left(\beta^{-M} \alpha^{M}\right),
\end{aligned}
$$

in $L^{2}$ norm for any $M \in \mathbb{N}$. Moreover, for a continuous function $\ell$, we have

$$
\ell u=\ell\left(x_{0}\right) \alpha^{1 / 2} \varphi\left(\alpha\left(x-x_{0}\right)\right) e^{i \beta x}+o_{\alpha \rightarrow \infty}(1),
$$

in $L^{2}$ norm. Using that $\left\|T(\ell)^{1 / 2}\right\| \leq\|\ell\|_{\infty}^{1 / 2}$, for all function $\ell \in L^{\infty}$ with $\ell \geq 0$, we get

$$
\begin{aligned}
T(f) T(g) v & =\operatorname{PfPgPu}=\operatorname{PfPgu}+\mathcal{O}\left(\alpha \beta^{-1}\right) \\
& =g\left(x_{0}\right) \operatorname{PfPu}+\mathcal{O}\left(\alpha \beta^{-1}\right)+o_{\alpha \rightarrow \infty}(1) \\
& =g\left(x_{0}\right) \operatorname{Pfu}+\mathcal{O}\left(\alpha \beta^{-1}\right)+o_{\alpha \rightarrow \infty}(1) \\
& =(f g)\left(x_{0}\right) P u+\mathcal{O}\left(\alpha \beta^{-1}\right)+o_{\alpha \rightarrow \infty}(1) \\
& =(f g)\left(x_{0}\right) v+\mathcal{O}\left(\alpha \beta^{-1}\right)+o_{\alpha \rightarrow \infty}(1) .
\end{aligned}
$$

Taking $\beta=\alpha^{2} \rightarrow+\infty,(7.6)$ implies $\|v\|=1+o_{\alpha \rightarrow \infty}(1)$. On the other hand, (7.8) leads to

$$
T(f) T(g) v=(f g)\left(x_{0}\right) v+o_{\alpha \rightarrow \infty}(1) .
$$

Then, $(f g)\left(x_{0}\right) \in \sigma(T(f) T(g))=\sigma\left(T(g)^{1 / 2} T(f) T(g)^{1 / 2}\right)$. Therefore,

$$
[\inf (f g), \sup (f g)] \subset \sigma\left(T(g)^{1 / 2} T(f) T(g)^{1 / 2}\right) .
$$


Recall that the essential spectrum of a self-adjoint bounded operator on an infinite Hilbert space is never empty. Therefore, if $\inf (f g)=\sup (f g),(7.5)$ implies the theorem.

Assume now that $\inf (f g)<\sup (f g)$. Then $[\inf (f g), \sup (f g)]$ is an interval with non empty interior. From the definition of the essential spectrum, this interval is necessarily inside the essential spectrum of $T(g)^{1 / 2} T(f) T(g)^{1 / 2}$. This achieves the proof of the second inclusion of Coburn's theorem.

Acknowledgment. The authors would like to thanks A. Böttcher for providing the reference of Roch and Silbermann. They also thank the anonymous referee for his careful reading of the paper.

\section{References}

1. B. Bercu, F. Gamboa, and A. Rouault, Large deviations for quadratic forms of stationary Gaussian processes, Stochastic Process. Appl. 71 (1997), no. 1, 75-90.

2. A. Böttcher, J. Gutiérrez-Gutiérrez, and P. Crespo, Mass concentration in quasicommutators of Toeplitz matrices, J. Comput. Appl. Math. 205 (2007), no. 1, 129-148.

3. A. Böttcher and B. Silbermann, Analysis of Toeplitz operators, second ed., Springer Monographs in Mathematics, Springer-Verlag, Berlin, 2006, Prepared jointly with Alexei Karlovich.

4. A. Böttcher and B. Silbermann, Introduction to large truncated Toeplitz matrices, Springer-Verlag, New York, 1999.

5. L.A. Coburn, The $C^{*}$-algebra generated by an isometry, Bull. Amer. Math. Soc. 73 (1967), 722-726.

6. W. Bryc and A. Dembo, Large deviations for quadratic functionals of Gaussian processes, J. Theoret. Probab. 10 (1997), no. 2, 307-332, Dedicated to Murray Rosenblatt.

7. A. Dembo and O. Zeitouni, Large deviations techniques and applications, second ed., Applications of Mathematics (New York), vol. 38, Springer-Verlag, New York, 1998.

8. J. Dereziński and C. Gérard, Scattering theory of classical and quantum N-particle systems, Texts and Monographs in Physics, Springer-Verlag, Berlin, 1997.

9. R. Gray, Toeplitz and circulant matrices: a review, Foundations and Trends in Communications and Information Theory 2 (2006), no. 3, 155-239.

10. U. Grenander and G. Szegö, Toeplitz forms and their applications, California Monographs in Mathematical Sciences, University of California Press, Berkeley, 1958.

11. T. Kato, Perturbation theory for linear operators, Classics in Mathematics, Springer-Verlag, Berlin, 1995, Reprint of the 1980 edition.

12. N. Nikolski, Operators, functions, and systems: an easy reading. Vol. 1, Mathematical Surveys and Monographs, vol. 92, American Mathematical Society, Providence, RI, 2002, Hardy, Hankel, and Toeplitz, Translated from the French by A. Hartmann.

13. M. Reed and B. Simon, Methods of modern mathematical physics. I, second ed., Academic Press Inc., New York, 1980, Functional analysis.

14. S. Roch and B. Silbermann, Limiting sets of eigenvalues and singular values of Toeplitz matrices, Asymptotic Analysis 8 (1994), 293-309.

15. S. Serra Capizzano, Distribution results on the algebra generated by Toeplitz sequences: a finite-dimensional approach, Linear Algebra Appl. 328 (2001), no. 1-3, 121-130.

16. H. Widom, Asymptotic behavior of block Toeplitz matrices and determinants. II, Advances in Math. 21 (1976), no. $1,1-29$. 